\title{
An Autofocus Approach for Residual Motion Errors with Application to Airborne Repeat-Pass SAR Interferometry
}

\author{
Karlus A. C. de Macedo, Rolf Scheiber and Alberto Moreira \\ Microwaves and Radar Institute \\ German Aerospace Center \\ Oberpfaffenhofen, Germany \\ e-mail: karlus.macedo@dlr.de
}

\begin{abstract}
Airborne repeat-pass SAR data are very sensible to sub-wavelength deviations from the reference track. To enable repeat-pass interferometry a high-precision navigation system is needed. Due to the limit of accuracy of such systems, deviations in the order of centimeters remain between the nominal and the processed reference track causing mainly undesirable phase undulations and misregistration in the interferograms, referred as residual motion errors. Up to now only interferometric approaches, as multi-squint, are used to estimate those deviations to compensate for such residuals. In this paper we present for the first time the use of the Autofocus technique for residual motion errors. A very robust autofocus technique has to be used since the accuracy of the estimated motion has to be at millimeter scale. Because we deal with low-altitude-stripmap mode data we propose a new robust autofocus technique based on the WLS (Weighted Least-Squares) phase estimation and Phase Curvature Autofocus (PCA) extended to the rangedependent case. We call this new technique WPCA (Weighted PCA). While the multi-squint approach is only able to estimate the baseline variation from coregistered images, the autofocus approach has the advantage of being able to estimate motion deviations independendtly for each image. Repeat-pass data of the E-SAR system of the German Aerospace Center (DLR) are used to demostrate the performance of the proposed approach.
\end{abstract}

\section{INTRODUCTION}

Deviations of the SAR antenna from the reference track cause mismatch between the echo signal and the reference azimuth chirp used for SAR focusing. This mismatch gives origin to phase errors in the signal history. These errros cause defocusing and target position errors in range and azimuth directions. Specially airborne systems are affected due to the flight instabilities. Such phase errors can be cancelled by measuring, through a navigation system, the deviations and subsequent removal with Motion Compensation (MoComp). These measurements have to be performed at subwavelength scale from pulse to pulse to obtain high-resolution SAR images. This puts an extreme burden on the navigation system. Autofocus techniques are used to estimate phase errors beyond the capability of the navigation system [1], [2].

With the use of modern navigation instrumentation, with precision in the order of centimeters, there is practically no defocusing on high-resolution SAR data and no need to use conventional autofocus. For very high-resolution data, broadening of the SAR impulse response function still affecting the resolution requiring basic autofocus [3].

Even when there is no relevant defocus in the SAR image we still have motion errors that cause mainly misregistration and undesirable phase undulations in the interferograms. These motion errors we shall refer as residual motion errors. For single-pass interferometry most of the residual motion errors cancel out due to the signal beating of the two interferometric channels, which have practically the same deviations. Differently, for repeat-pass interferometry the channels have uncorrelated motion errrors causing undesired phase artefacts in the final interferogram, [4], [5], [6].

To cope with the recent demands of airborne repeat-pass interferometry and its applications, such as differential interferometry (D-InSAR), which measures terrain movements at millimeter scale, it is necessary to estimate phase errors with accuracy under 0.25 radians. This requirement motivated the development of algorithms to estimate phase errors from interferometric data, such as multi-squint [6]. Multi-squint has shown to be robust with the improvement of coherence and decrease of phase errros in interferograms. The multi-squint approach is only able to estimate the baseline variations from coregistered images. An autofocus approach has the advantage of being able to estimate phase errors independently for each image not requiring any interferometric processing.

Up to now, an autofocus approach with algorithms such as Map Drift [1], or Phase Gradiente Autofocus (PGA) [2] were not used for residual motion errors. This is because that requires further refinements on autofocus. We propose a new robust autofocus technique based on the WLS (Weighted Least-Squares) phase estimation [7] and on the Phase Curvature Autofocus (PCA) [8] extended to range-dependent case. We call this new technique WPCA (Weighted PCA).

Section II presents the new autofocus technique for residual motion errors (WPCA). In Section III, repeat-pass data at L-band of the E-SAR system of the German Aerospace Center (DLR) are used to demonstrate the performance of the proposed WPCA algorithm. In section IV we address the conclusions. 


\section{THE ALGORITHM FOR RESIDUAL MOTION ERRORS}

\section{A. The WPCA kernel}

Due to the not wide use of the PCA algorithm [8], only one kernel (non-range dependent), for the maximum-likelihood (ML) estimator, has been derived and used for phase doubledifference estimation up to now [10]

$$
\hat{\tilde{\phi}}_{e}^{M L}(l)=\arg \sum_{k=1}^{K}\left[g_{k, l-1}\left(g_{k, l+1}^{*}\right)^{2} g_{k, l+1}\right] .
$$

where $g_{i}$ is the decompressed and deramped azimuth windowed signal in the $k^{\text {th }}$ range bin. This kernel has poor results and increase of noise in double-differentiation process due to the ML formulation and shift operations. Besides it does not use a range dependent geometry model. Some estimation difficults were reported in [9], [10].

Other algorithms use phase gradient (initially proposed for spotlight) for strip-map images as the PWE-PGA algorithm [11]. It converts blocks of the SAR strip into spotlight domain. It has poor results for residual motion due to artefacts introduced in the block conversion and discotinuities between blocks that can reach several centimeters deteriorating residual motion estimations. Additionaly, the PWE-PGA uses a ML formulation for the weights, which assumes white Gaussian noise (WGN) clutter. The PMA and SPGA [12] are other solutions to avoid double-differentation. They use a Doppler centroid estimation to handle with the unknown linear components. This estimation is inaccurate to sub-pixel shifts of each target.

We propose an algorithm based on the PCA frame work combined with a better target selection and an optimal, in the WLS sense, range-dependent phase kernel. We call this algorithm WPCA (Weighted Least-Squares Phase Curvature Autofocus). We start by deriving the WPCA estimation kernel. First we take

$$
\ddot{g}(t)=\frac{\partial^{2}|g(t)| \exp [-j \phi(t)]}{\partial^{2} t},
$$

where $\phi(t)$ the phase of the azimuth windowed signal $g(t)$. Since we use the WLS formulation, we do not need to include the amplitude of the $g(t)$ signal to solve for $\ddot{\phi}(t)$, as in the case of the PGA kernel. In fact, the amplitude of $g(t)$ does not affect the gradient computation and it is a good idea to force its amplitude to be equal one. Solving the above equation for $\ddot{\phi}(t)$ and $|g(t)|=1$ we get

$$
\ddot{\phi}(t)_{k}=\left.\operatorname{Im}\left[\ddot{g}_{k}(t) g_{k}^{*}(t)+\dot{g}_{k}(t) \dot{g}_{k}^{*}(t)\right]\right|_{|g(t)|=1},
$$

where $\ddot{\phi}(t)_{k}$ is an estimate or realization in the $k^{t h}$ range bin. Then, the WPCA kernel for the phase double-derivative becomes

$$
\hat{\tilde{\boldsymbol{\Phi}}}_{y z}=\left(\mathbf{A}^{T} \mathbf{W}^{-1} \mathbf{A}\right)^{-1} \mathbf{A}^{T} \mathbf{W} \ddot{\boldsymbol{\Phi}}
$$

with

$$
\ddot{\boldsymbol{\Phi}}=\left[\begin{array}{c}
\ddot{\phi}_{1} \\
\vdots \\
\ddot{\phi}_{K}
\end{array}\right], k=1,2,3 \ldots K,
$$

and

$$
\hat{\tilde{\mathbf{D}}}_{y z}=\left[\begin{array}{l}
\hat{\ddot{D}}_{y} \\
\hat{\ddot{D}}_{z}
\end{array}\right]=\frac{\lambda}{4 \pi} \hat{\tilde{\boldsymbol{\Phi}}}_{y z}=\frac{\lambda}{4 \pi}\left[\begin{array}{l}
\hat{\ddot{\phi}}_{y} \\
\hat{\ddot{\phi}}_{z}
\end{array}\right],
$$

where $\hat{\overline{\mathbf{\Phi}}}_{y z}$ is the matrix with the estimated horizontal $\hat{\ddot{\phi}}_{y}$ and vertical $\ddot{\phi}_{z}$ double-derivative of the phase errors components, respectively, $\hat{\overline{\mathbf{D}}}_{y z}$ is the matrix with the horizontal $\hat{\ddot{D}}_{y}$ and vertical $\hat{\ddot{D}}_{z}$ acceleration of the motion deviations from the reference track, respectively. The $\mathbf{W}$ is the diagonal matrix with the weight values based on the signal-to-clutter ratio (SCR) estimations according to [7]

$$
w=1 / \sigma_{\nu}^{2} \approx 1 /\left((1 / 2) R+(5 / 24) R^{2}\right),
$$

where $R$ is the inverse of the SCR and is given by

$$
R \approx \frac{1}{d}\left(4\left(2 c^{2}-d\right)-4 c \sqrt{4 c^{2}-3 d}\right),
$$

with

$$
\begin{gathered}
d=E[|g(t)|], \\
c=E\left[|g(t)|^{2}\right],
\end{gathered}
$$

where $E[\cdot]$ is the expectation operator.

The matrix $\mathbf{A}$ is the range-dependet model [11]

$$
\mathbf{A}=\left[\begin{array}{cc} 
\pm \sin \theta_{1} & \cos \theta_{1} \\
\vdots & \vdots \\
\pm \sin \theta_{K} & \cos \theta_{K}
\end{array}\right]
$$

\section{B. Filter to avoid SCR degradation}

Derivative operations work as a high-pass filter and therefore degradates the SCR due to the increase of the clutter phase undulations. To avoid increase of white noise clutter, we compute the phase double-derivatives using a numerical differentiation techniques, differently from the kernel in (1), wich uses shift operations. Nevertheless the main problem persists since in practice we have non-white noise. In order to optimal use the WLS formulation, in which the weights are computed according to the phase variance with (7)-(10), we have to keep the variance of the phase double-derivative in the same levels as before the differentiations. We do this by applying to $\hat{\ddot{\phi}}_{k}$ an Autoregressive Moving Average (ARMA) to filter the clutter. This filter removes also part of the residual motion error frequency within the synthetic aperture. Thus, the filter length should be chosen according to the expected frequency of the motion error within the aperture and to the clutter power spectrum.

For residual motion errors coming from the inaccuracy of modern navigation systems, we can assume that the motion deviations from the nominal track are almost linear or slightly quadratic within an aperture of about $90 \mathrm{~m}$ according to [5]. 


\section{The WPCA frame work}

We implement a target pre-selection criteria that includes all pixels of the SAR images above a certain amplitude threshold, $T_{A}$. In a rectangular moving window of $\Omega_{r g}$ and $\Omega_{a z}$ dimension in range and azimuth, respectively, we take the maximum amplitude pixel. The next step is to window around this maximum pixel adaptatively in a azimuth window with dimension $\Omega$ defined at $-10 \mathrm{~dB}$ relative to the maximum. These targets are chosen as possible candidates. Then, each target candidate is decompressed and deramped as in the PCA frame work [8].

The double-derivative estimates are computed using (3), and their weights according to (7)-(10). Targets with weights less than a certain value $T_{w}$, or alternatively SCR less than $T_{s c r}$, are discarded. The reason is to avoid wrong SCR estimations, since [7] shows that (7)-(10) are valid for values greater than $1 \mathrm{~dB}$.

Each smoothed $\ddot{\phi}_{k}$ estimate is aligned according to their azimuth bin. To avoid undesired artefacts, coming from transient response of the phase of the windowed signal, the borders of each phase estimate are discarded. We use half of the vector length. Note that each vector $\ddot{\phi}_{k}$ corresponds to a synthetic aperture length.

Finally, the acceleration of the motion errors are estimated using the WPCA kernel, followed by double-integration to determine the estimated motion deviations. Due to the unknown initial conditions $\Phi(0)$ and $\dot{\Phi}(0)$, we remove the global linear component and offset after the phase integration by fitting it to a first order polynomial.

\section{Accuracy and Convergence}

Let assume that the variance of the smoothed doublederivative phase estimates are approximately equal to the phase variance of the estimates given by (7)-(10). In this case the covariance matrix of the phase estimation process is

$$
\mathbf{C}_{\hat{\mathbf{\Phi}}_{\mathbf{y z}}} \approx\left(\mathbf{A}^{T} \mathbf{W}^{-1} \mathbf{A}\right)^{-1}=\left[\begin{array}{ll}
C_{00} & C_{01} \\
C_{10} & C_{11}
\end{array}\right]
$$

Furthermore, we assume that the number of estimates are large and diverse enough so that the bias of the acceleration estimations can be considered zero or constant along the azimuth bins. After the double-integration, if the strip is long, a small constant bias may result in a quadratic curvature with amplitude greater than the desired accuracy. In this case we make the mean value of the motion estimations along azimuth equal zero. This procedure removes the constat bias but also a possible global quadratic component of the motion error. But, for long strips it is very unlikely to have a relevant global quadratic term in the motion error.

After motion compensation, the WPCA is repeated iteratively, to refine the estimations, until the maximum of the norm $\left\|\hat{\boldsymbol{\Phi}}_{u}-\hat{\mathbf{\Phi}}_{u-1}\right\|$, between iterations $u$, is equal or less than a tolerance value, Tol. When this condition is satisfied the WPCA achieved convergence. The tolerance can be defined by being the sum of a absolute tolerance $\mathrm{Tol}_{a}$ and a relative tolerance $\mathrm{Tol}_{r}$. The relative tolerance should be greater or

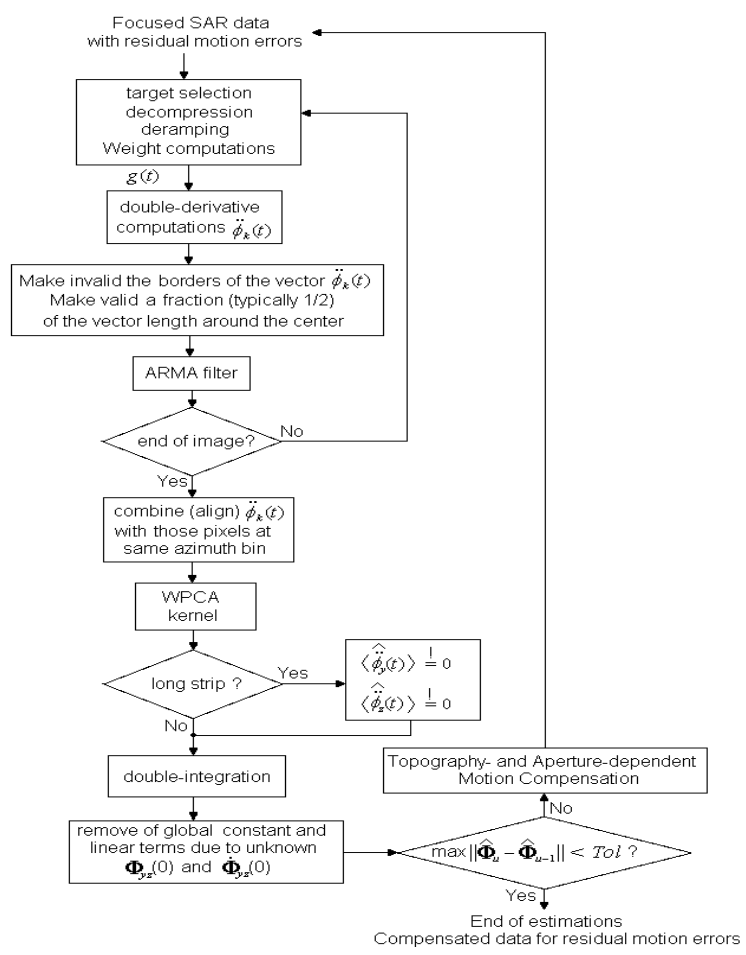

Fig. 1. WPCA algorithm block diagram.

equal the theorical phase accuracy times a value $\beta_{\phi}$. Thus, Tol can be defined as

$$
T o l=\beta_{\phi} \sqrt{C_{00}+C_{11}}+T_{o l} .
$$

The $\beta_{\phi}$ helps the algorithm to be robust against procedural errors that are not modeled by the assumptions.

Fig. 1 shows the block diagram of the WPCA algorithm.

\section{RESUlTS}

To demonstrate the performance and effectiveness of the WPCA algorithm for residual motion errros we use airborne repeat-pass data of the E-SAR system (DLR). This system uses an up-to-date high precision navigation system. For processing we used the Extended Chirp Scaling followed by the Precise Topography- and Aperture dependent motion compensation, PTA-MoComp [13]. Thus, the errors that persist in the images and interferograms are mainly residual motion errors due to the insufficiencies of the navigation system.

We apply the WPCA algorithm on the SAR image of Oberpfaffenhofen area, Germany, which is mostly agricultural with some man-made areas and practically flat (see Fig. 2(a)). This SAR image is a SLC (Single Look Complex) at L-band with $2.5 \mathrm{~km} \times 4.0 \mathrm{~km}$ (range $\mathrm{x}$ azimuth) dimensions.

We focus now on interferometric data. The images were coregistered according only to the nominal baselines. Fig. 2(b) shows the coherence histograms. Fig. 2(c) shows the residual interferogram, i.e. interferogram minus DEM (Digital Elevation Model), before WPCA and Fig. 2(d) after applying WPCA. There is a clear improvement in the accuracy when using the WPCA. The interferograms are scaled from $[-\pi, \pi]$. 


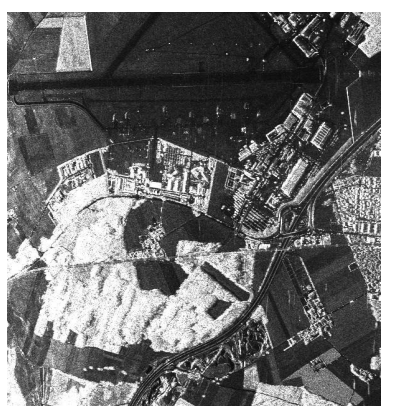

(a) amplitude image

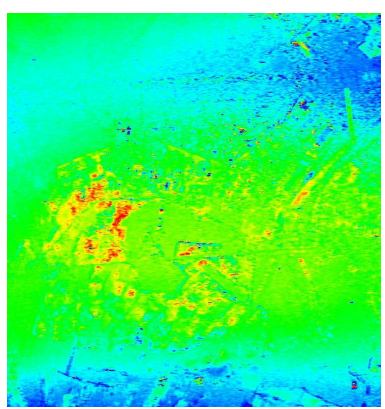

(c) residual interf. before WPCA (b) coherence histogram

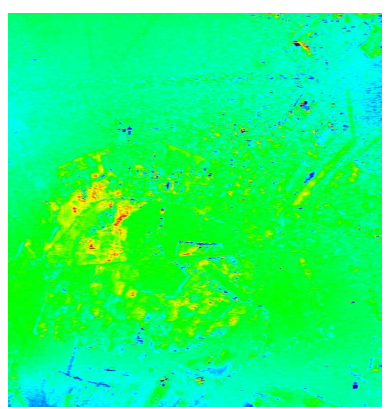

(d) residual interf. after WPCA

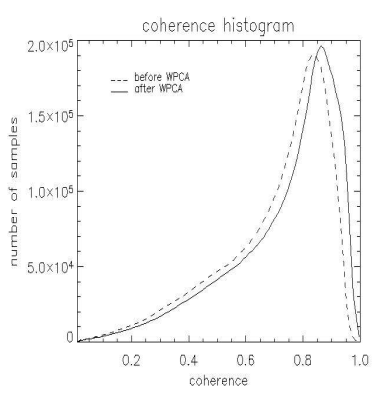

Fig. 2. L-band results, Oberpfaffenhofen.

In both interferograms, the remained global linear and offset terms of the baseline were removed by fitting the residual interferogram into a linear model as in [6]. This could be also done with corner reflectors strategically placed. The corrected interferogram contains phase artefacts from DEM errors.

Fig. 3 shows the residual motion estimations from WPCA. Fig. 3(a) and (b) are the horizontal and vertical motion deviations of the master image, respectively, and in Fig. 3(c) and (d) the same for the slave image. The convergence is achieved at the second iteration, for the master, and at the third iteration, for the slave, with about $0.05 \mathrm{~cm}(0.25$ radians $)$ accuracy.

\section{CONCLUSION}

We have proposed a new and robust autofocus algorithm the so-called WPCA. We demonstrated that it is capabale of estimating residual motion erros from strip-map SAR data. The use of an autofocus approach for residual motion errors opens the possibility of performing airborne differential SAR interferometry (D-InSAR) with millimeter accuracy for long temporal baselines since it does not require any interferometric processing for the estimation. Further work consists in testing the algorithm for different images, bands and baselines.

\section{ACKNOWLEDGMENT}

The authors would like to thank Mennato Catillo for the initial investigations on PGA algorithms.

\section{REFERENCES}

[1] C.E. Mancill and J.W. Swiger, A map drift autofocus technique for correcting higher order SAR phase errors, Proc. 27th Annual Tri-Service Radar Symposium Record, pp. 523-25, Monterey, CA., Jun., 1981

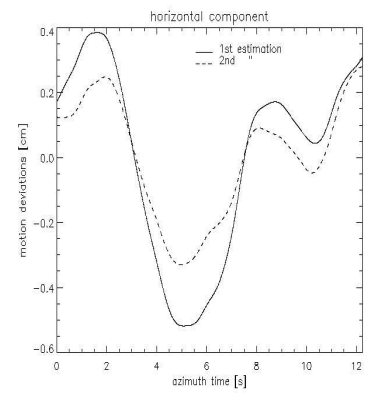

(a) horizontal dev., master

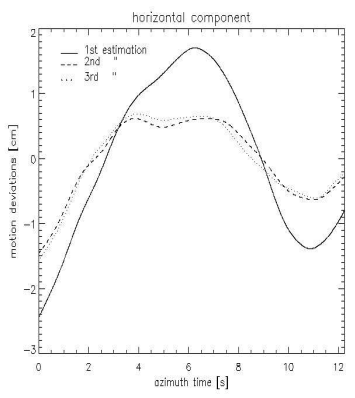

(c) horizontal dev., slave

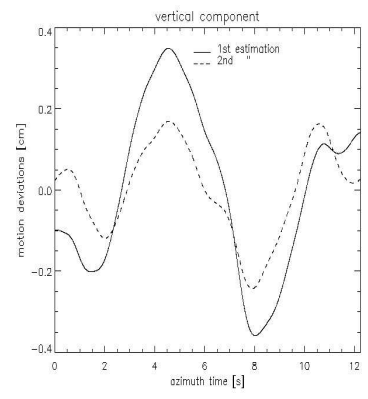

(b) vertical dev., master

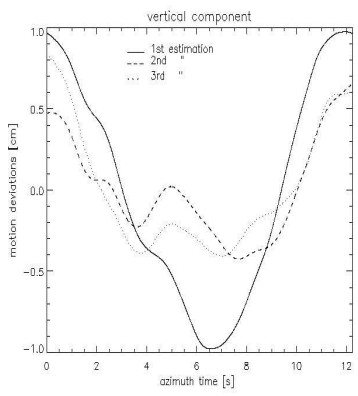

(d) vertical dev., slave
Fig. 3. Motion Estimations.

[2] D.E. Wahl, P.H. Eichel, D.C. Ghiglia and C.V. Jakowatz Jr., Phase gradient autofocus - a robust tool for high resolution SAR phase correction, IEEE Trans. Aerosp. and Eletron. Syst., Vol. 30, No. 3, pp. 837-835, Oct. 1994.

[3] H. Cantalloube and P. Dubois-Fernandez, Airborne X-band SAR Image with $10 \mathrm{~cm}$ resolution: technical challenges and preliminary results, IEE Proc. Radar Sonar Navig., Vol. 153, No. 2, pp. 163-176, April 2006.

[4] D.R. Stevens, I.G. Cumming and A.L. Gray, Options for Airborne Interferometric SAR Motion Compensation, IEEE Trans. Geosci. and Remote Sensing, Vol. 33, No. 2, pp. 409-419, March, 1995.

[5] G. Fornaro, G. Francheschetti and S. Perna", Motion Compensation Errors: Effects on the Accuracy of Airborne SAR Images, IEEE Trans. Aerosp. Electron. Syst., Vol. 41, No. 4, pp. 1338-1352, Oct., 2005.

[6] A. Reigber, P. Prats and J.J. Mallorqui, Refined estimation of timevarying baseline errors in airborne SAR Interferometry, IEEE Trans. Geosci. and Remote Sesing Lett., Vol. 3, No. 1, pp. 145-149, Jan., 2006.

[7] H.L. Ye, T.S. Yeo and Z. Bao, Weighted least-squares estimations of phase erros for SAR/ISAR autofocus, IEEE Trans. Geosci. Remote Sensing, Vol. 37, No. 5, pp. 2487-2494, Sep., 1999.

[8] D.E. Wahl, C.V. Jakowatz Jr. and P.A. Thompson, New approach to strip-map SAR autofocus, Proc. VI IEEE Digital Signal Processing Workshop, pp. 53-56, Josemite, CA, Oct. 1994.

[9] T.J. Sutton, S.A. Chapman and H.D. Griffiths, Robustness and effectiveness of autofocus algorithms applied to diverse seabed environments, Proc. V ECUA: European Conference on Underwater Acoustics, pp. 407412, Lyon, France, Jul., 2000.

[10] J.H. Callow, Signal processing for synthetic aperture sonar image enhacement, $\quad \mathrm{PhD}$ Thesis, Department of Eletrical and Eletronic Enginnering, University of Canterburry, New Zealand, 2003

[11] G.T. Douglas, J.S. Bates, D.V. Arnold, D.G. Long and A. Robertson, Range dependent phase gradient autofocus, Proc. IGARSS'98, Seatle, WA., Jul., 1998.

[12] M.P. Hayes, J.H. Callow and P.T. Gough, Strip-map phase gradient autofocus, Proc. IV Image and Vision Computing New Zealand, pp. 71-76, Auckland, New Zealand, Dec., 2002

[13] K.A.C. de Macedo and R. Scheiber, Precise Topography-and ApertureDependent Motion Compensation for Airborne SAR, IEEE Trans. Geosci. and Remote Sesing Lett., Vol. 2, No. 2, pp. 172-176”, Apr., 2005 . 